\title{
Controle por Busca Extremal de Robôs Móveis Planares *
}

\author{
Jean A. Tomé* Alessandro J. Peixoto** \\ * Laboratório de Engenharia de Controle e Automação, Universidade \\ Federaldo Rio de Janeiro, RJ, (e-mail: jamerico@poli.ufrj.br). \\ ** Departamento de Engenharia Eletrônica e de Computação, \\ Universidade Federal do Rio de Janeiro, RJ, (e-mail: \\ jacoud@poli.ufrj.br).
}

\begin{abstract}
This paper presents a practical application of an extremum seeking control (ESC) based on a periodic perturbation with the objective of tracking a source inserted in the planar mobile robot working plane. The strategy is based on two control loops: one internal loop for tracking a periodic trajectory and an external loop based on ESC. The internal tracking control provides the periodic disturbance signals to the external control (ESC) which generates desired references for the internal control to track the source. Two different experiments are conducted: the robot tracking a fixed and a moving source.

Resumo: Este artigo apresenta a aplicação prática de um controle por busca extremal (ESC) baseado em perturbação periódica com o objetivo de rastrear uma fonte inserida virtualmente no plano de trabalho de robôs móveis planares. A estratégia proposta é baseada em duas malhas: uma interna para rastreamento de uma trajetória periódica e uma malha externa baseada em ESC. O controle interno de rastreamento utilizado fornece os sinais de perturbação periódica para o controle externo (ESC) e este gera referências de rastreamento para o controle interno seguir a fonte. Dois diferentes experimentos são conduzidos: o robô rastreando a fonte parada e em movimento.
\end{abstract}

Keywords: Extremum Seeking Control, Source Seeking, Computer Vision, Mobile Robotics, 2DoF.

Palavras-chaves: Busca Extremal, Rastreamento de Fonte, Visão Computacional, Robótica Móvel, 2DoF.

\section{INTRODUÇÃO}

O Controle para Busca Extremal, ou Extremum Seeking Control (ESC), é uma técnica de controle muito utilizada em otimização em tempo real onde há um objetivo a ser minimizado (maximizado) que é função do estado de um sistema dinâmico incerto. Os mais populares algoritmos para otimização sem restrição utilizam informação do gradiente da função objetivo em questão. Devido as incertezas, o ESC não utiliza-se informação do gradiente da função objetivo que é muitas vezes não acessível em tempo de execução.

Como descrito em Tan et al. (2010), o ESC encontrou aplicações desde desde: esquemas de controle que mantivesse máxima transferência de potência entre uma linha de transmissão e um bonde elétrico, passando por otimização de um motor de combustão interna (DRAPER (1951)) até o projeto de freios ABS com em Yu and Ozguner (2002), onde é realizado a otimização do coeficiente de força de fricção que depende do terreno em que o carro se encontra.

Em particular, para robótica móvel, o ESC fornece uma poderosa ferramenta para projeto de sistemas de controle

\footnotetext{
* Fundação de Amparo à Pesquisa do Estado do Rio de Janeiro (FAPERJ) pela apoio financeiro ao desenvolvimento desse projeto.
}

que independam dos mecanismos de localização usuais, tais como GPS e câmera (Cochran et al. (2008)), bastando apenas que um sinal detectável emitido por uma fonte seja captado pelo robô. Em Zhang et al. (2007b) e Zhang et al. (2007a) são desenvolvidos ESCs baseados em função excitação periódica em que os sinais de excitação são injetados com compensações adequadas de fase e ruídos no sistema são considerados em Liu and Krstic (2010).

Em TOMÉ et al. (2016) foi desenvolvida uma malha de ESC com o objetivo de rastrear um alvo por meio da maximização de área captada do mesmo através de visão computacional, onde apenas um grau de liberdade foi considerado (1DoF). Essa abordagem foi bem sucedida e provocou o desenvolvimento do trabalho atual, com a utilização do ESC para o rastreamento de fonte por robôs móveis terrestres em um plano de trabalho, consolidando uma otimização em dois graus de liberdade (2DoF).

Neste trabalho é proposta uma estratégia de controle baseada em duas malhas: uma interna para rastreamento de uma circunferência (trajetória periódica em geral) de raio constante e centro variável, sendo que o centro é gerado pela malha externa baseada em controle por busca extremal (ESC). A malha externa é projetada para assegurar que o centro evolua no tempo e convirja para o maxi- 
mizante da função custo em questão. A malha interna é baseada em um controle proporcional não-linear de posição (linear e angular) largamente encontrado na literatura, como em Siegwart and Nourbakhsh (1997).

A principal contribuição encontra-se em utilizar trajetórias periódicas para o robô que fornecem duas vantagens: (i) os sinais de ditter de Ariyur and Krstic (2003), Zhang et al. (2007b) e Zhang et al. (2007a) necessários para o esquema do controle por busca extremal utilizado na malha externa são gerados automaticamente com as devidas defasagens de fase necessárias, devido ao desacoplamento natural do movimento circular imposto ao uniciclo e (ii) do ponto de vista prático e experimental, evita-se mudança no sentido de rotação das rodas do uniciclo, gerando uma trajetória mais suave uma vez que a região da zona morta do motores não é atingida pois os motores não passam por velocidade zero.

\section{FORMULAÇÃO DO PROBLEMA}

Para o caso em que as rodas/atuadores são idênticos, as acelerações angulares das rodas e/ou momento de inércia do conjunto motor/roda e constante de tempo elétrica dos motores são desprezíveis, considera-se o seguinte modelo para o uniciclo:

$$
\begin{aligned}
m \dot{u} & =-\frac{2 k^{2}}{r^{2} R} u+\frac{k}{r R} u_{t}, \\
\dot{x} & =\cos (\theta) u, \\
\dot{y} & =\operatorname{sen}(\theta) u, \\
\frac{J}{l} \dot{\omega} & =-\frac{2 k^{2} l}{r^{2} R} \omega+\frac{k}{r R} u_{r}, \\
\dot{\theta} & =\omega .
\end{aligned}
$$

sendo $R$ a resistência da armadura dos motores, $k$ a correspondente constante de torque, $r$ é o raio das rodas, $l$ é a distância entre os eixos das rodas, $m$ é a massa do veículo, $J$ é o momento de inércia do veículo com respeito ao eixo vertical passando pelo C.G., $(x, y)$ é a posição do C.G. do veículo, $u$ é a componente da velocidade linear do C.G. na direção das rodas, $\omega$ é velocidade angular do veículo e $u_{t}=v_{1}+v_{2}$ e $u_{r}=v_{1}-v_{2}$ são os sinais de controle de translação e rotação, respectivamente, com $v_{1}$ e $v_{2}$ as tensões de armadura dos motores.

O Objetivo de Controle é otimizar em tempo real a função $f(x, y)$ que representa a medição, por exemplo, de temperatura que apresenta um único máximo no ambiente de trabalho do veículo na posição $\left(x^{\star}, y^{\star}\right)$. Deseja-se projetar uma lei de controle $u_{t}$ e $u_{r}$ tal que o C.G. do veículo descrito pelas equações dinâmicas (1) atinja o maximizante $\left(x^{\star}, y^{\star}\right)$ e permaneça no seu em torno o mais próximo possível.

Considere que a função de intensidade do sinal captado (ou função custo) no plano do trabalho do robô possui forma a quadrática:

$$
h(x, y)=h^{\star}-h^{\prime \prime}\left(\left(x-x^{\star}\right)^{2}+\left(y-y^{\star}\right)^{2}\right),
$$

sendo $h^{\prime \prime}>0$. Essa função apresenta um máximo global $h^{\star}$ em $x=x^{\star}$ e $y=y^{\star}$, que corresponde a posição da fonte. O problema consiste em realizar estimativas de $x^{\star}$ e $y^{\star}$ em tempo real, considerando o desconhecimento dos parâmetros $h^{\star}, x^{\star}, y^{\star}$ e $h^{\prime \prime}$. Deseja-se que as estimativas $(\hat{x}$ e $\hat{y})$ para os maximizantes $\left(x^{\star}, y^{\star}\right)$ sejam cada vez mais próximas de $x^{\star}$ e $y^{\star}$, elevando o valor da função intensidade até sua estabilização em torno do seu valor máximo.

Além disso, valia-se experimentalmente também o desempenho do esquema de controle quando existe movimentação da fonte emissora do sinal, implicando que $x^{\star}$ e $y^{\star}$ definem funções do tempo.

A solução proposta baseia-se na implementação de duas malhas de controle: uma malha interna para rastreamento de trajetória periódica e uma malha externa baseada em ESC para atingir o objetivo de controle.

\section{MALHA INTERNA: RASTREAMENTO}

Considere o modelo dinâmico do robô (1), tendo como sinal de entrada $u_{t}$ (soma das tensão nas rodas) e $u_{r}$ (diferença de tensão nas rodas). Seja $\left(x_{r e f}, y_{r e f}\right)$ a posição desejada o C.G. $(x, y)$ e $R_{r}$ o raio de uma circunferência centrada em $\left(x_{r e f}, y_{r e f}\right)$. O ângulo entre o vetor formado do C.G. para a posição desejada $\left(x_{r e f}, y_{r e f}\right)$ e a orientação do robô (vetor que aponta no sentido do eixo $x$ do robô) é denotado por $\theta_{\text {aux }}$, ver Figura 1. Com o objetivo de assegurar que o robô siga a tangente da circunferência com centro em $\left(x_{r e f}, y_{\text {ref }}\right)$ e raio $R_{r}$, defina o erro angular $\theta_{d}=\theta_{\text {aux }}-\pi / 2$ e sejam ainda $\Delta_{x}:=x_{r e f}-x$ e $\Delta_{y}:=y_{r e f}-y$ os desvios na posição do C.G..

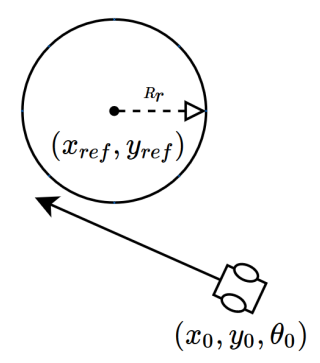

Figura 1. Desenho esquemático do robô sendo controlado para seguir uma referência circular em torno de $\left(x_{r e f}, y_{\text {ref }}\right)$ com raio $R_{r}$.

A lei de controle Siegwart and Nourbakhsh (1997)

$$
\begin{aligned}
& u_{t}=U, \\
& u_{r}=-k_{1} U L-k_{2}|U| \tilde{\theta}-\frac{U \cos (\tilde{\theta})}{L+R_{r}} .
\end{aligned}
$$

assegura rastreamento da circunferência com centro em $\left(x_{r e f}, y_{\text {ref }}\right)$ e raio $R_{r}$, sendo $\tilde{\theta}:=\theta-\theta_{d}, D:=\sqrt{\Delta_{x}^{2}+\Delta_{y}^{2}}$ e $L:=D-R_{r}$ representa o erro entre a posição do C.G. do robô e o ponto sobre a circunferência alinhado com a direção formada pelo C.G. e o centro da circunferência (erro linear). Os ganhos $k_{1}, k_{2}>0$ são constantes de projeto e o sinal de controle $u_{t}=U$ é assumido constante por simplicidade. $\mathrm{O}$ controle possui três termos e cabe um comentário sobre eles:

- $k_{1} U L$ : termo responsável por reduzir o erro linear aproximando o robô da circunferência. 
- $k_{2}|U| \tilde{\theta}$ : termo responsável por reduzir o erro angular e alinhar o robô na direção da tangente a circunferência. - $\frac{U \cos (\tilde{\theta})}{L+R_{r}}$ : de dentro do círculo $(L<0)$. Este termo possui relevância apenas quando $D=L+R_{r} \approx 0$.

Os ganhos $k_{1}, k_{2}$ e o valor de $U$ são obtidos visando garantir que o erro de rastreamento do robô em torno da referência seja aceitável quando $\left(x_{r e f}, y_{r e f}\right)$ variar ao longo do tempo. Note que, para o objetivo deste trabalho rastreamento com muita precisão não é necessário. O fundamental é que o robô realize movimento periódicos em geral para resultar em uma bom aproveitamento dos sinais na malha externa do controle por busca extremal.

\section{MALHA EXTERNA: OTIMIZAÇÃO}

Para otimizar (2) é necessário obter estimativas para o gradiente da função $h(\cdot)$ com componentes $\frac{\partial h}{\partial x}$ e $\frac{\partial h}{\partial y}$.

A abordagem aqui explorada para a estimativa dessas componentes utiliza o desacoplamento natural entre as componentes de posição do C.G. $(x$ e $y)$ do robô quando este executa o movimento circular imposto pela malha interna, que resulta em duas senoides defasadas de $\pm 90^{\circ}$.

Com essas senoides, o ESC baseado em perturbação periódica de Ariyur and Krstic (2003) dispensa a necessidade de um sinal de perturbação externo.

A malha completa de controle contendo a malha interna, a planta e malha externa é apresentada na Figura 2, de forma que $y_{n}=\left(a_{n}, b_{n}\right)_{w}^{\phi_{n}}$ denota uma senoide do tipo $y(t)=a_{n} \operatorname{sen}\left(\omega t+\phi_{n}\right)+b_{n}$, para $n=\{1,2, \ldots, 6\}$, sendo $a_{n}, b_{n}, \phi_{n}$ e $\omega$ constantes. O bloco $C(\cdot)$ constitui o controle da malha interna de rastreamento do robô. Note que a malha externa fornece o centro da circunferência a ser rastreada pela malha interna. Admite-se que o tempo de estabilização do robô em torno de uma nova referência é muito menor que a taxa de variação entre uma referência e outra.

\subsection{Funcionamento Básico do Esquema de Controle}

Considere que o controle $C(\cdot)$, ao receber uma referência $\left(x_{r e f}, y_{r e f}\right)$, a posição $\left(x_{0}, y_{0}\right)$ e orientação $\theta_{0}$ do robô forneça o sinal de controle $u_{r}(t)$ com $u_{t}=U$ constante, capaz de fazê-lo circular ao redor dessa referência.

Desconsiderando-se o transitório, a posição do robô em regime permanente é descrita por

$$
\begin{gathered}
x_{1}=a_{x 1} \operatorname{sen}\left(\omega t+\phi_{x 1}\right)+b_{x 1}, \\
y_{1}=a_{y 1} \operatorname{sen}\left(\omega t+\phi_{y 1}\right)+b_{y 1},
\end{gathered}
$$

As senóides em (5) entram como argumento da função custo não linear $h(\cdot)$. Utilizando os primeiros termos da série de Taylor, tem-se a seguinte aproximação: $z_{1}=$ $h\left(x_{1}, y_{1}\right) \approx h\left(b_{x 1}, b_{y 1}\right)+\frac{\partial h}{\partial x}\left(b_{x 1}, b_{y 1}\right) a_{x 1} \operatorname{sen}\left(\omega t+\phi_{x 1}\right)+$ $\frac{\partial h}{\partial y}\left(b_{x 1}, b_{y 1}\right) a_{y 1} \operatorname{sen}\left(\omega t+\phi_{y 1}\right)$, que podem ser representados por

$$
z_{1}=a_{z x} \operatorname{sen}\left(\omega t+\phi_{x 1}\right)+a_{z y} \operatorname{sen}\left(\omega t+\phi_{y 1}\right)+b_{z},
$$

sendo $a_{z x}:=\frac{\partial h}{\partial x}\left(b_{x 1}, b_{y 1}\right), a_{z y}:=\frac{\partial h}{\partial y}\left(b_{x 1}, b_{y 1}\right)$ e $b_{z}:=$ $h\left(b_{x 1}, b_{y 1}\right)$.
O filtro passa alta da Figura 2 é construído na forma: 1 $\frac{\omega_{L}}{s+\omega_{L}}=\frac{s}{s+\omega_{L}}$. O termo (6) é entrada desse filtro passa alta, utilizado para eliminar o nível médio $b_{z}$, cuja saída é dada por $y_{4}=a_{z x} \operatorname{sen}\left(\omega t+\phi_{x 1}\right)+a_{z y} \operatorname{sen}\left(\omega t+\phi_{y 1}\right)+b_{z}-$ $a_{z x} k_{L} \operatorname{sen}\left(\omega t+\phi_{x 1}+\phi_{H}\right)-a_{z y} k_{L} \operatorname{sen}\left(\omega t+\phi_{y 1}+\phi_{H}\right)-b_{z}+\pi$, sendo $k_{L}=\frac{w_{L}}{\sqrt{w_{L}^{2}+w^{2}}}$ o ganho do filtro passa baixa na frequência $\omega$ e $\pi$ termos exponenciais decrescentes.

Agora, escolhendo $x_{0 L}=b_{z}-\frac{a_{z y} \omega_{L}\left(\omega \cos \phi-\omega_{L} \sin \phi\right)}{w_{L}^{2}+w^{2}}-$ $\frac{a_{z x} \omega_{L}\left(\omega \cos \phi-\omega_{L} \sin \phi\right)}{w_{L}^{2}+w^{2}}$ como condição inicial do filtro passa baixa, elimina-se os termos exponenciais do transitório. Considerando que o ganho do filtro passa baixa $k_{L}$ é muito menor que $a_{z y}$ e $a_{z x}$, tem-se que:

$$
z_{2}=a_{z x} \operatorname{sen}\left(\omega t+\phi_{x 1}\right)+a_{z y} \operatorname{sen}\left(\omega t+\phi_{y 1}\right) .
$$

Um procedimento análogo pode ser aplicado a posição do robô descrita em (5), removendo o valor médio de $x_{1}$ e $y_{1}$ e obtendo-se:

$$
\begin{aligned}
& x_{2}=a_{x 2} \operatorname{sen}\left(\omega t+\phi_{x 2}\right), \\
& y_{2}=a_{y 2} \operatorname{sen}\left(\omega t+\phi_{y 2}\right) .
\end{aligned}
$$

Além disso, considere ainda as aproximações: $a_{x 2} \approx a_{x 1}$, $a_{y 2} \approx a_{y 1}, \phi_{x 2} \approx \phi_{x 1}$ e $\phi_{y 2} \approx \phi_{y 1}$. As senóides $x_{2} \mathrm{e}$ $y_{2}$ resultantes da filtragem da posição do robô (8) são multiplicadas pela senóide resultante da filtragem da saída da função custo em (7), podendo escrever que:

$$
\begin{aligned}
& x_{3}=a_{x 1} \operatorname{sen}\left(\omega t+\phi_{x 1}\right)\left(a_{z x} \operatorname{sen}\left(\omega t+\phi_{x 1}\right)+a_{z y} \operatorname{sen}\left(\omega t+\phi_{y 1}\right)\right) \\
& y_{3}=a_{y 1} \operatorname{sen}\left(\omega t+\phi_{y 1}\right)\left(a_{z x} \operatorname{sen}\left(\omega t+\phi_{x 1}\right)+a_{z y} \operatorname{sen}\left(\omega t+\phi_{y 1}\right)\right)
\end{aligned}
$$

O resultado desse produto pode ser representado através de transformações trigonométricas:

$$
\begin{aligned}
x_{3} & =a_{x 1} a_{z x} \frac{\cos \left(\phi_{x 1}-\phi_{x 1}\right)}{2}-\frac{a_{x 1} a_{z x}}{2} \cos \left(2 \omega t+\phi_{x 1}+\phi_{x 1}\right) \\
& +a_{x 1} a_{z y} \frac{\cos \left(\phi_{x 1}-\phi_{y 1}\right)}{2}-\frac{a_{x 1} a_{z y}}{2} \cos \left(2 \omega t+\phi_{x 1}+\phi_{y 1}\right), \\
y_{3} & =a_{y 1} a_{z x} \frac{\cos \left(\phi_{y 1}-\phi_{x 1}\right)}{2}-\frac{a_{y 1} a_{z x}}{2} \cos \left(2 \omega t+\phi_{y 1}+\phi_{x 1}\right) \\
& +a_{y 1} a_{z y} \frac{\cos \left(\phi_{y 1}-\phi_{y 1}\right)}{2}-\frac{a_{x 1} a_{z y}}{2} \cos \left(2 \omega t+\phi_{y 1}+\phi_{y 1}\right) .
\end{aligned}
$$

Note que pela natureza circular do movimento as posições $x$ e $y$ do robô (8) estão defasadas de $90^{\circ}$, de forma que $\phi_{x 1}=\phi_{y 1} \pm 90^{\circ}$, resultando em $\cos \left(\phi_{x 1}-\phi_{y 1}\right)=\cos \left(\phi_{y 1}-\right.$ $\left.\phi_{x 1}\right)=0$. Aplicando essa propriedade e simplificando 9 , resulta em:

$$
\begin{array}{r}
x_{3}=a_{x 1} a_{z x} \frac{1}{2}-\frac{a_{x 1} a_{z x}}{2} \cos \left(2 \omega t+\phi_{x 1}+\phi_{x 1}\right)- \\
\frac{a_{x 1} a_{z y}}{2} \cos \left(2 \omega t+\phi_{x 1}+\phi_{y 1}\right), \\
y_{3}=a_{y 1} a_{z y} \frac{1}{2}-\frac{a_{y 1} a_{z x}}{2} \cos \left(2 \omega t+\phi_{y 1}+\phi_{x 1}\right)- \\
\frac{a_{y 1} a_{z y}}{2} \cos \left(2 \omega t+\phi_{y 1}+\phi_{y 1}\right) .
\end{array}
$$

Os termos em (10) são entradas de um filtro passa baixas na forma $\frac{\omega_{L}}{s+\omega_{L}}$ com correspondentes saídas: 


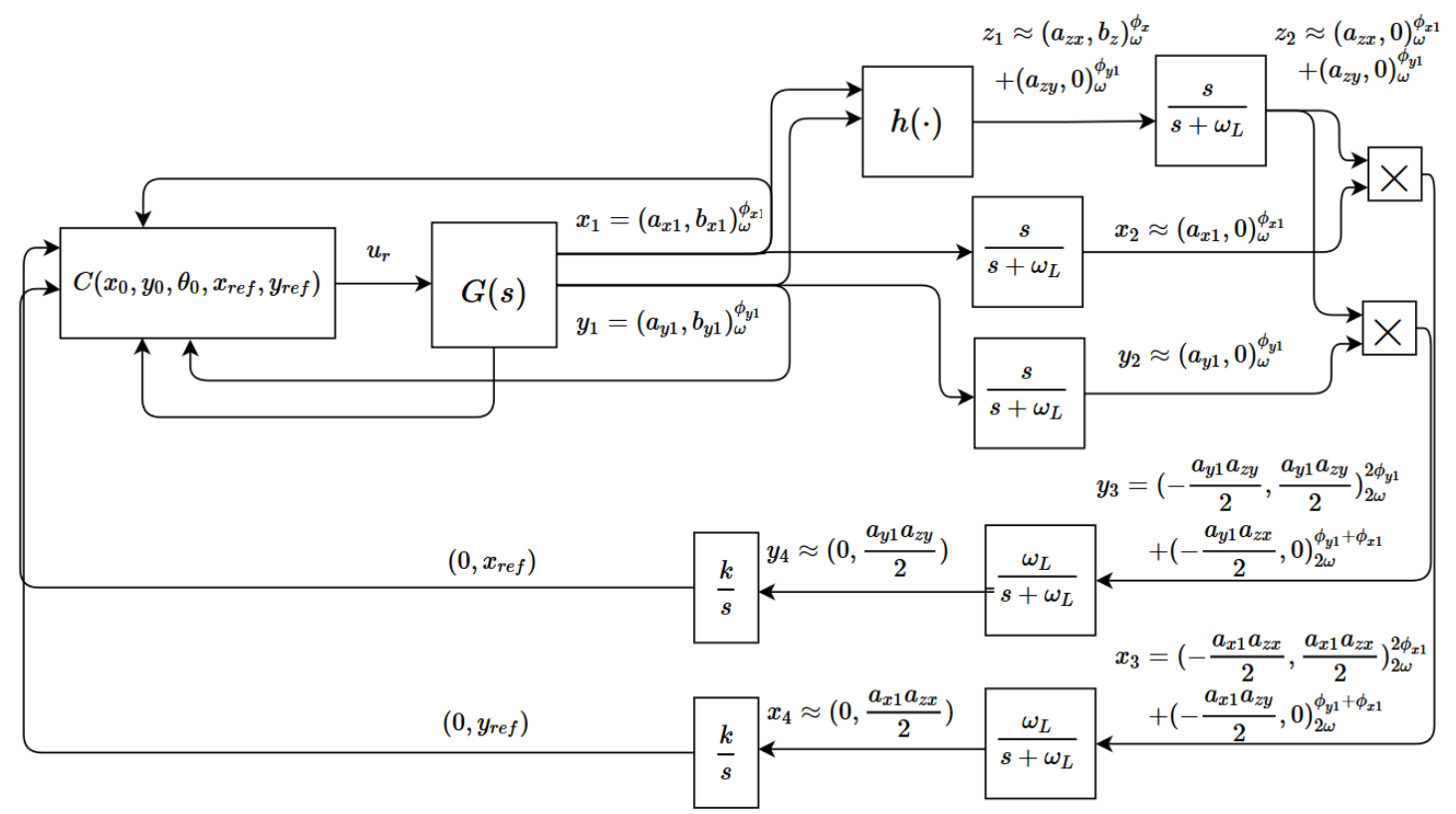

Figura 2. Visão geral da malha fechada incluindo o controle interno, planta e a malha externa (ESC).

$$
\begin{array}{r}
x_{4}=a_{x 1} a_{z x} \frac{1}{2}-K_{L} \frac{a_{x 1} a_{z x}}{2} \cos \left(2 \omega t+\phi_{x 1}+\phi_{x 1}\right) \\
-K_{L} \frac{a_{x 1} a_{z y}}{2} \cos \left(2 \omega t+\phi_{x 1}+\phi_{y 1}\right)+\pi \\
y_{4}=a_{y 1} a_{z y} \frac{1}{2}-K_{L} \frac{a_{y 1} a_{z x}}{2} \cos \left(2 \omega t+\phi_{y 1}+\phi_{x 1}\right) \\
-K_{L} \frac{a_{y 1} a_{z y}}{2} \cos \left(2 \omega t+\phi_{y 1}+\phi_{y 1}\right)+\pi .
\end{array}
$$

Sendo $\pi$ o termo do transitório. Para as condições inicial dos filtros iguais a:

$$
\begin{array}{r}
X_{0 L}=a_{x 1} a_{z x} \frac{1}{2}-\frac{a_{x 1} a_{z x} w_{L}\left(2 w \cos \phi_{4}-\omega_{L} \sin \phi_{4}\right)}{4 w^{2}+w_{L}^{2}}- \\
X_{0 L}=a_{y 1} a_{z y} \frac{1}{2}-\frac{a_{x 1} a_{z y} w_{L}\left(2 w \cos \phi_{4}-\omega_{L} \sin \phi_{4}\right)}{4 w^{2}+w_{L}^{2}} \\
\frac{a_{y 1} a_{z y} w_{L}\left(2 w \cos \phi_{4}-\omega_{L} \sin \phi_{4}\right)}{4 w^{2}+w_{L}^{2}}-
\end{array}
$$

e com os ganho do filtro passa baixa $k_{L}=\frac{\omega_{L}}{\sqrt{4 \omega^{2}+\omega_{L}^{2}}} \approx$ 0, tem-se como saída:

$$
\begin{aligned}
& x_{5}=a_{x 1} a_{z x} \frac{1}{2}, \\
& y_{5}=a_{y 1} a_{z y} \frac{1}{2} .
\end{aligned}
$$

Finalmente, tem-se os integradores com entradas $x_{5}$ e $y_{5}$ $\left(x_{4}\right.$ e $y_{4}$ filtrados, ver Figura 2) e saídas $x_{\text {ref }}$ e $y_{r e f}$. Dessa forma, compõe-se o novo par referência $\left(x_{r e f}, y_{\text {ref }}\right)$ para o controle $C(\cdot)$.

A análise de estabilidade rigorosa por ser obtida utilizando a Análise de Média (Averaging Analysis) Ariyur and Krstic (2003) e será omitida neste trabalho para poupar espaço.

\section{RESULTADOS EXPERIMENTAIS}

A bancada experimental é composta por um computador, um par de de módulos de comunicação $X B E E$, um robô móvel terrestre do modelo uniciclo $3 \mathrm{Pi}$ da Polulu, um campo para testes e uma câmera (webcam). A Figura 3 apresenta a imagem do robô com um par de cores identificadoras sobre sua superfície no campo. Através do uso da

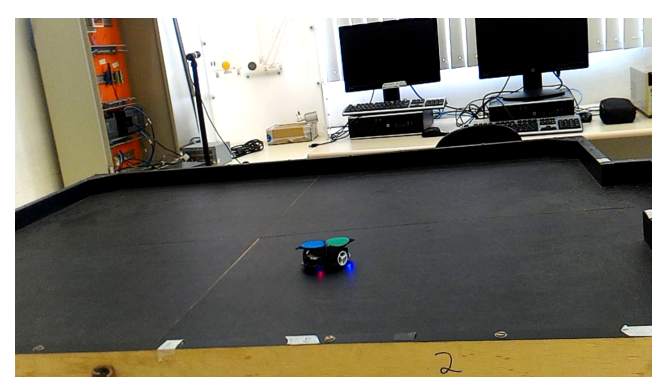

Figura 3. Robô com um par de cores identificadoras sobre sua superfície no campo disponível para testes.

webcam, situada em um suporte elevado sobre o campo, a imagem do espaço de trabalho e do robô (identificado com um par de cores distintas sobre sua superfície) é capturada e enviada ao computador em ambiente Windows que executa um algoritmo de processamento de imagem.

O sinal de controle é calculado e enviado para o robô através dos módulos de comunicação $X B E E$ via porta serial (USB). A comunicação é via rádio frequência e utiliza o padrão IEEE 802.15.4.

O controlador do robô recebe a mensagem também através de um módulo Xbee e converte a mensagem em um sinal PWM gerando os níveis de tensão (0 e 5V) para cada roda. 


\subsection{Robô localizando a fonte}

Para o primeiro experimento considerou-se que o robô, inicialmente em movimento circular ao redor da origem, $\left(x_{r 0}, y_{r 0}\right)$, deseja localizar uma fonte em seu espaço de trabalho. A malha de busca extremal é iniciada, gerando a cada instante uma nova referência $\left(x_{r e f}, y_{r e f}\right)$ para o controlador do robô. Para esse experimento, o robô é inicializado em $x_{r 0}=60, y_{r 0}=88$. A fonte está localizada em $(166,212)$, fisicamente no extremo oposto do campo disponível em laboratório.

A Figura 4 apresenta uma visão geral do movimento do robô ao longo do campo de bancada. Destaca-se o movimento do robô durante a inicialização em preto, aguardando-se estabilização em torno de $(60,88)$. Em seguida, o algoritmo de ESC é inicializado, e a trajetória ao longo do espaço de trabalho é mostrada em azul. Notase que o ESC foi capaz de levar o robô até a fonte. Através

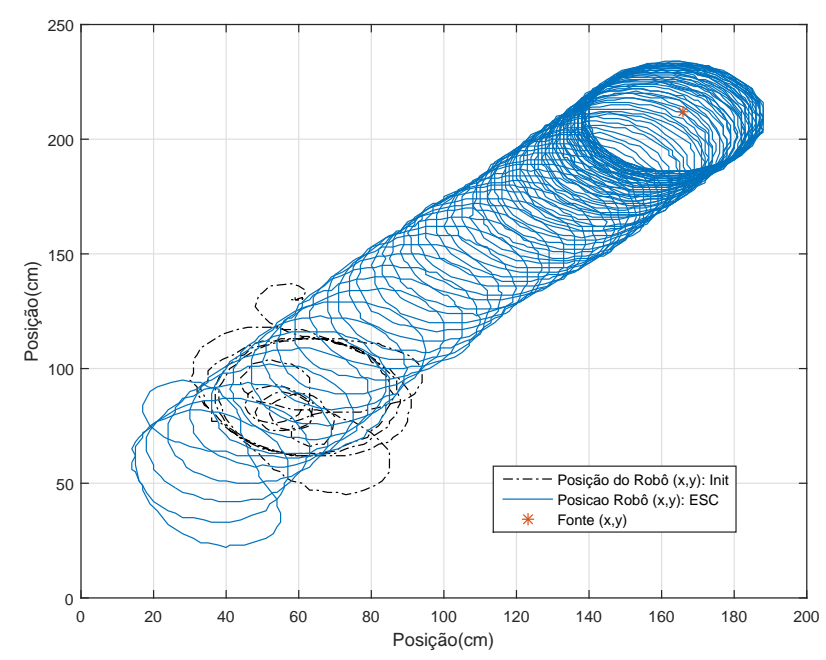

Figura 4. Visão geral do movimento do robô ao longo do espaço do trabalho em busca da fonte.

da Figura 5(a), pode-se observar a evolução temporal do valor da função custo durante o experimento, destacandose quatro intervalos: (i) durante a inicialização, entre $t=0$ e $t \approx 5 s$; (ii) entre $t \approx 5 s$ e $t=12 s$, quando o robô está estabilizado e girando em torno de $(60,88)$, e a função custo tem valor médio de -26000; (iii) no intervalo transitório entre $t \approx 12 \mathrm{~s}$ e $t=120 \mathrm{~s}$, em que o robô inicia o movimento em direção ao sentido de aumento da função custo; (iv) Após $t=120 \mathrm{~s}$, em que o robô inicia seu movimento de estabilização tendendo ao valor máximo (400), fazendo a função custo oscilar com valor médio de aproximadamente (200).

A Figura 5(b) mostra a evolução da posição do robô discriminada ao longo do tempo em comparação com a posição da fonte. Até $t \approx 5 s$, a inicialização leva o robô ao valor médio de $(60,88)$, oscilando com amplitude de $\approx 25 \mathrm{~cm}$. Entre $t \approx 5 \mathrm{~s}$ e $t=12 \mathrm{~s}$, o robô se encontra estabilizado em torno de $(60,88)$. Após $t=12 s$, ele inicia o movimento de elevação de seu valor médio em direção da fonte.
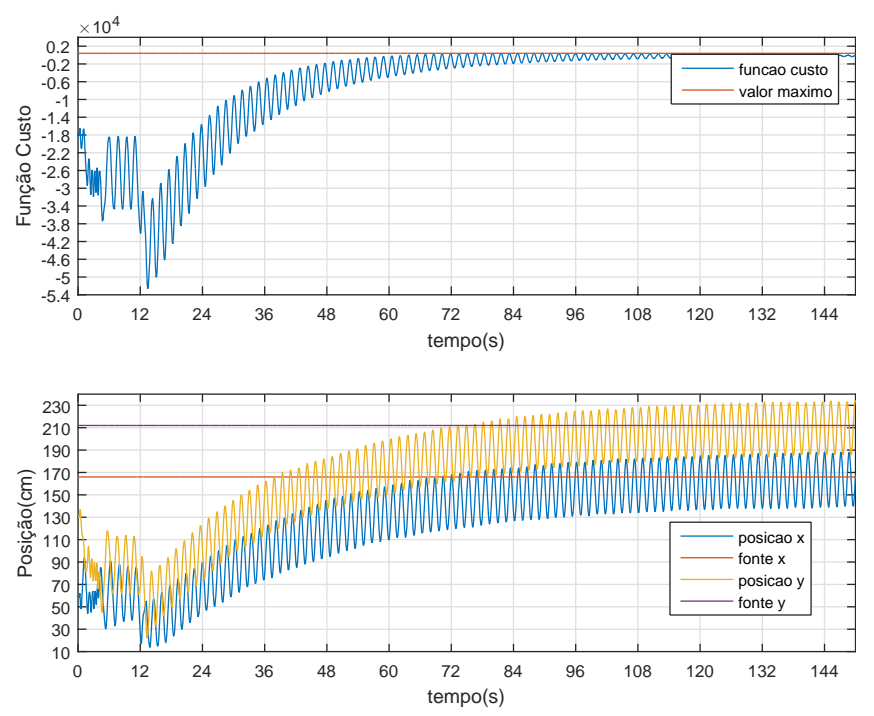

Figura 5. (a) Função custo ao longo do tempo e (b) Posição do robô $(x, y)$ em comparação com a fonte.

\subsection{Robô perseguindo a fonte}

No segundo experimento considerou-se o caso em que o robô inicialmente se encontra girando ao redor da fonte. A fonte então inicia um movimento circular ao longo de seu espaço de trabalho, fazendo com que a função custo tenha um novo par maximizante $\left(x^{\star}, y^{\star}\right)$ a cada instante. A malha de busca extremal é iniciada, gerando um novo par referência $\left(x_{r e f}, y_{r e f}\right)$ para o controlador do robô.

É importante salientar que nesse caso há uma competição entre a malha de controle da ESC e a velocidade de deslocamento da fonte. Assim, para perseguir fontes que se movem rapidamente, deve-se diminuir os coeficientes dos filtros da malha da ESC (Figura 2), correndo o risco de instabilidade, e aumentar os ganhos do controle de rastreamento do robô. Nesse experimento considerou-se que a fonte se move lentamente, descrevendo a seguinte trajetória de $x_{\text {ref }}=119+70 \sin (0.04 t)$ e $y_{\text {ref }}=226+$ $70 \cos (0.04 t)$.

A Figura 6 apresenta uma visão geral do movimento do robô ao longo da bancada. Destaca-se o movimento do robô durante a inicialização em preto, aguardandose estabilização em torno de $(119,226)$. Em seguida, o algoritmo de ESC é inicializado, e sua trajetória ao longo do espaço de trabalho é apresentada em azul. A trajetória da fonte é apresentada em vermelho. Observa-se que o ESC foi capaz de fazer o robô perseguir a fonte. Na Figura 7(a), pode-se observar quatro intervalos na evolução temporal do valor da função custo durante o teste: (i) durante a inicialização, entre o $t=0 \mathrm{~s}$ e $t \approx 10 \mathrm{~s}$, em que o robô se moveu em direção a fonte em $(119,226)$; (ii) entre $t=10 \mathrm{~s}$ e $t \approx 18 \mathrm{~s}$, quando o robô está estabilizado e girando em torno da fonte, fazendo com que a função custo permanecesse em cerca de -150 , em uma região próxima de seu valor máximo. (iii) de $t=18 \mathrm{~s}$ em diante, a fonte iniciou seu movimento ao longo do espaço de trabalho e a ESC começou a guiar o robô a fim de persegui-lá. Após os $60 \mathrm{~s}$, a variação da função custo permanece entre 0 e -500 , significando que o robô conseguiu manter uma distância fixa - e próxima - da fonte. A Figura 7 (b) 


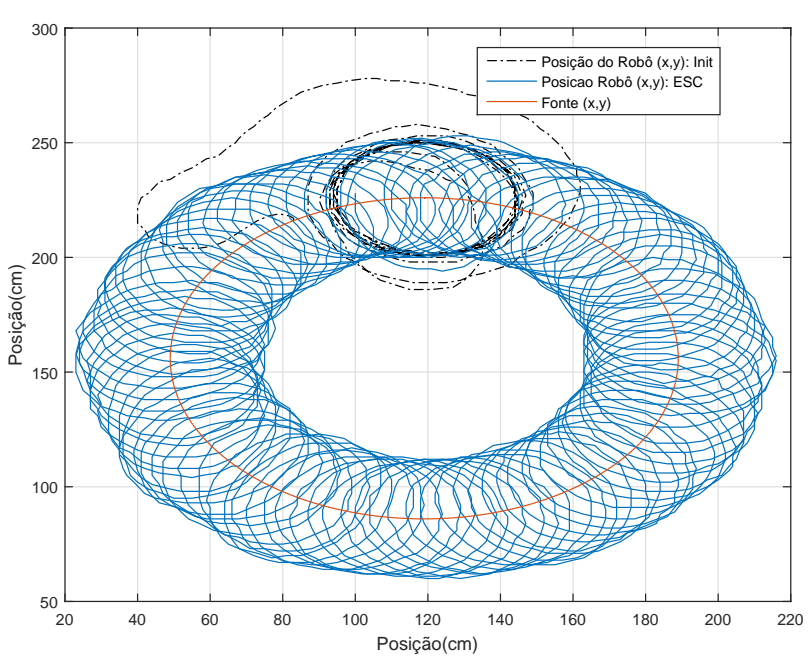

Figura 6. Visão geral do movimento do robô ao longo do espaço do trabalho em busca da fonte em movimento.
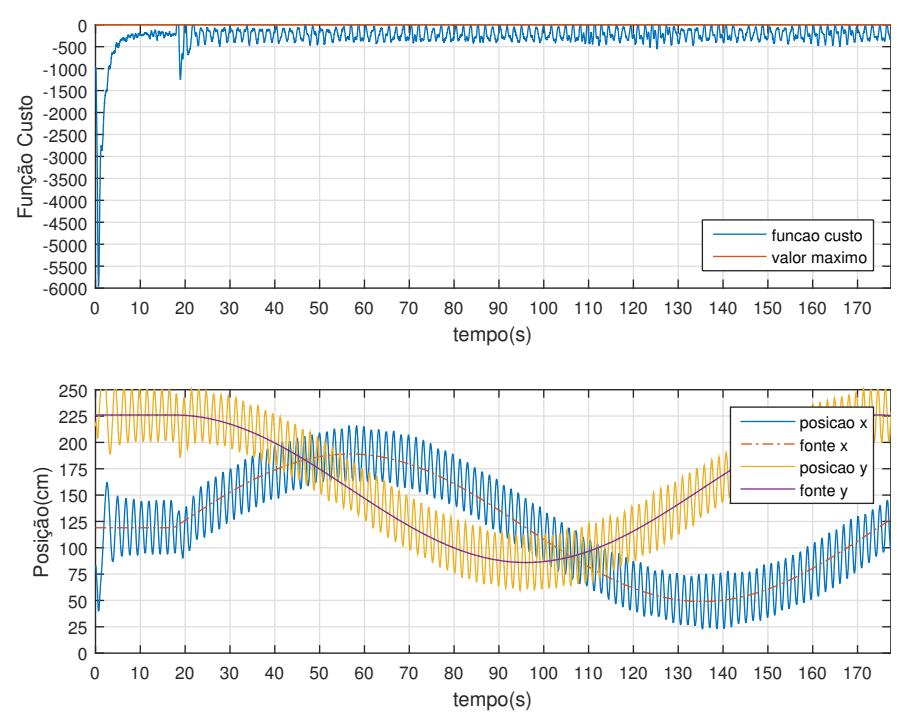

Figura 7. (a) Função custo ao longo do tempo e (b) Posição do robô $(\mathrm{X}, \mathrm{Y})$ em comparação com a fonte em movimento.

mostra a evolução da posição do robô discriminada ao longo do tempo em comparação com a posição da fonte. Até $t \approx 5 s$, a inicialização leva o robô ao valor médio de $(119,226)$. Entre $t \approx 5 s$ e $t=18 s$, o robô se encontra estabilizado e oscilando com amplitude de $\approx 25^{c} \mathrm{~m}$ em torno de $(119,226)$. Após $t=18 \mathrm{~s}$, ele inicia o movimento de perseguição da fonte ao longo do espaço de trabalho.

\section{CONCLUSÕES}

Este trabalho considerou o rastreamento de um fonte inserida virtualmente no plano de trabalho de robôs móveis planares. A estratégia considerada foi unir duas malhas de controle: uma interna para rastreamento de uma trajetória periódica e uma malha externa baseada em ESC. Enquanto a malha externa fornece sinais de referência para a malha interna rastrear, esta por sua vez fornece os sinais de perturbação periódica para o controle por busca extremal utilizado na malha externa. Este esquema permitiu evitar que os motores troquem o sentido de rotação e, com isso, evitem a zona morta do motor e resulte em trajetória mais suaves no plano de trabalho. Dois diferentes experimentos foram conduzidos: o robô rastreando a fonte parada e em movimento.

Com os resultados obtidos foi possível concluir que o controle para busca extremal aplicado à localização da fonte no espaço de trabalho mostrou-se bem sucedido, levando o robô até a posição real da fonte e fazendo com que o valor da função custo tendesse ao seu valor máximo (400). Todavia, o efeito dos filtros do sistema se mostraram não-desprezíveis, de forma que nos primeiros segundos da inicialização do ESC a referência gerada resulta num sentido contrário ao da fonte.

Durante todo os experimentos, o sinal de controle $u_{r}$ (diferença de tensão nas rodas) permaneceu em um patamar aceitável assim como o sinal de controle $u_{t}$ (soma de tensão nas rodinhas) que foi utilizado constante. Além disso, com a fonte em movimento, o esquema proposto apresentou bons resultados, estabilizando a perseguição um erro menor que $2 \mathrm{~cm}$. Como trabalhos futuros, destacam-se: realizar a análise de estabilidade completa, considerar esquemas mais robustos para rastreamento na malha interna e realizar uma sintonia da malha atual para maximizar o desempenho.

\section{AGRADECIMENTOS}

O presente trabalho foi realizado com apoio da Coordenação de Aperfeiçoamento de Pessoal de Nível Superior - Brasil (CAPES) Código de Financiamento 001.

\section{REFERÊNCIAS}

Ariyur, K.B. and Krstic, M. (2003). Real-Time Optimization by Extremum-Seeking Control.

Cochran, J., Siranosian, A., Ghods, N., and Krstic, M. (2008). Gps denied source seeking for underactuated autonomous vehicles in 3d. In Proc. IEEE Conf. on Robotics and Automation, 6985-6990. Pasadena.

DRAPER, C., L.Y. (1951). Principles of optimalizing control systems and an application to the internal combustion engine. American Society of Mechanical Engineers.

Liu, S.J. and Krstic, M. (2010). Stochastic nonholonomic source seeking. In Proc. IEEE Conf. on Decision and Control, 69856990. Atlanta.

Siegwart, R. and Nourbakhsh, I.R. (1997). Introduction to Autonomous Mobile Robots.

Tan, Y., Moase, W.H., Manzie, C., Nešić, D., and Mareels, I.M.Y. (2010). Extremum seeking from 1922 to 2010. In Proceedings of the 29th Chinese Control Conference, 14-26.

TOMÉ, J.A., MATTOS, G.P.A., Peixoto, A.J., and DIAS, D.P. (2016). Controle por busca extremal via visão computacional. Congresso Brasileiro de Automática.

$\mathrm{Yu}, \mathrm{H}$. and Ozguner, U. (2002). Extremum-seeking control strategy for abs system with time delay. In Proceedings of the 2002 American Control Conference (IEEE Cat. No.CH37301), volume 5, 3753-3758 vol.5. doi:10.1109/ACC.2002.1024511.

Zhang, C., Arnold, D., Ghods, N., Siranosian, A., and Krstic, M. (2007a). Source seeking with non-holonomic unicycle without position measurement and with tuning of forward velocity. Systems E control letters, 56, 245-252.

Zhang, C., Siranosian, A., and Krstic, M. (2007b). Extremum seeking for moderately unstable systems and for autonomous vehicle target tracking without position measurements. AUT, 43, 1832-1839. 